\title{
Novel carbon nanotube based three terminal devices
}

\author{
Stephen M. Lyth, L. D. Filip*, D.C. Cox, S. R. P. Silva \\ Nano-Electronics Centre, Advanced Technology Institute, University of Surrey, \\ Guildford, Surrey, GU2 7XH, United Kingdom \\ *1.filip@surrey.ac.uk \\ Telephone: +44 1481 686083, Fax: +44 1483689404
}

Carbon nanotubes since their discovery have created much industrial and scientific interest. Their unique properties in electrical conduction, chemical robustness and the ability to control the growth of aligned CNT films makes them an ideal candidate for high intensity electron sources. Combining these properties with that ability to solubilise CNTs within an aqueous solution further extends the application fields associated with these unique materials.

In this work we will make use of an acid oxidised carbon nanotube ink that has been developed [1] to provide a simple route for controlling threshold fields and the current density in a three terminal device configuration.

The nanotube ink developed in this research project has enabled us to demonstrate threshold fields as low as $0.8 \mathrm{~V} / \mu \mathrm{m}$, by tailoring the underlying substrate morphology.

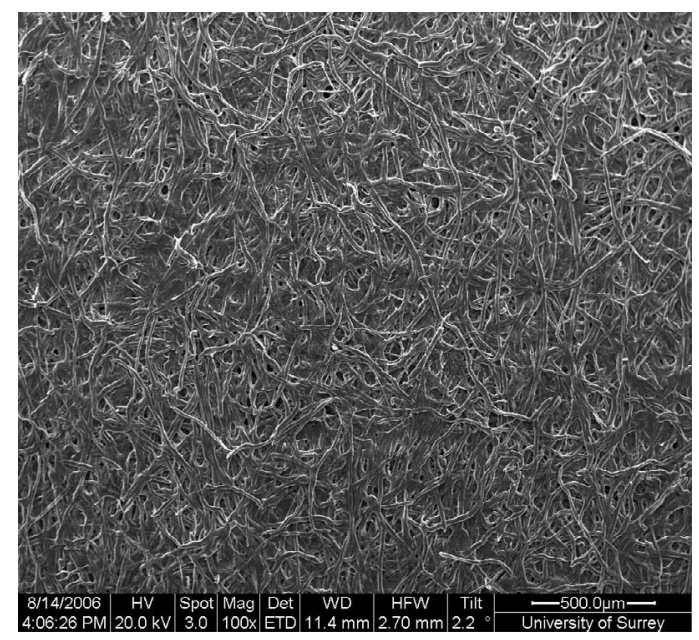

Figure 1

Furthermore, lithium functionalisation via a wet-chemistry technique has been used to tailor the work function of MWCNTs resulting in even lower threshold fields of 0.25 $\mathrm{V} / \mu \mathrm{m}[2]$. The inks produced can be inexpensively deposited over large areas (ink jet printing) on a variety of substrates (e.g.: paper, plastic, glass, silicon, etc.).

The $\mathrm{Li}^{+}$acid oxidised carbon nanotube ink has been dropped on a piece of filter paper, roughly $1 \mathrm{~cm}^{2}$ area and it was then placed to dry on a hot plate for 10 minutes in order to evaporate the water. The result was a dark region on the filter paper where the nanotubes formed a conducting mat, as can be seen in Figure 1. Choosing the filter paper as the support substrate for the carbon nanotube solution allows us to easily integrate it in a flexible three terminal device, as will be described.

The three-terminal device was made using a $3 \times 3 \mathrm{~cm}^{2}$ laminating pouch which had the sheet thickness of $125 \mu \mathrm{m}$. One of the sheets of the pouch was covered by a layer of $100 \mathrm{~nm}$ of DC sputtered Au. After the deposition of the Au, a matrix of 4x4 holes was made using a pin to punch through the plastic. Also a channel was cut in the Au covered sheet to allow the contacting of the nanotube mat. The paper covered with the nanotube mat was placed inside the pouch and then laminated using a conventional office laminator. The resulting structure is sketched in Figure 2.

Due to the crude manufacturing technique used, only two out of 4 devices could be used for FE testing mainly because of the way the holes were made. The plastic tended 
to cover the holes during the laminating process rendering the structure useless as the emitter would be completely covered.

The field emission tests were performed inside a vacuum chamber at a pressure of $10^{-6} \mathrm{mbar}$ in a triode like configuration as follows. The carbon nanotube mat was contacted through the channel previously cut, using silver paste and it was grounded. The Au gate electrode was kept at a fixed Figure 2

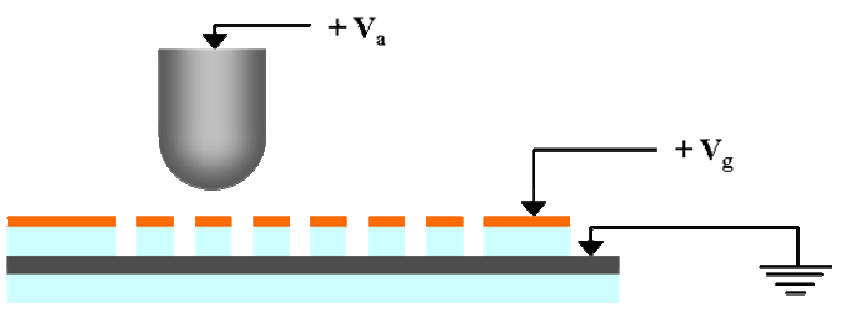

$125 \mu \mathrm{m}$ thick plastic

Filter paper soaked in CNT ink

$100 \mathrm{~nm}$ thick Au gate electrode deposited by DC sputtering

Spherical anode (diameter of the sphere is much bigger than the diameter of the holes) potential $V_{g}$ while the spherical anode placed at $300 \mu \mathrm{m}$ distance from the surface had a potential $V_{a}$ which was varied in $30 \mathrm{~V}$ steps. The data was recorded with the use of a computer and plotted in Figure 3 for various gate voltages. As it can be seen for the first two gate voltages there was no significant current registered at the

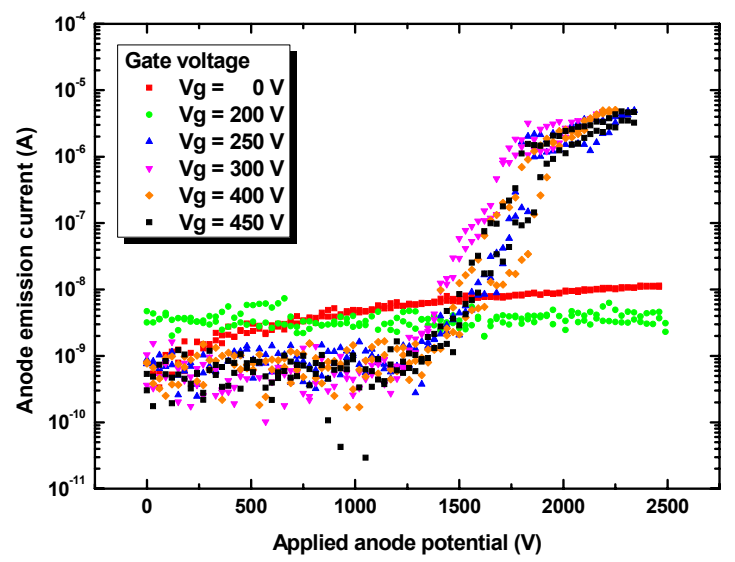

Figure 3

basis for new generation of flat panel displays. anode. For the rest of the applied potentials normal FE curves can be seen with a peak current of $5 \mu \mathrm{A}$. In the light of the method used to manufacture this device the results can only be seen as proof of concept and intense research is currently under was to improve the device.

It can be concluded that flexible FE devices can be manufactured using a cheap process. These devices can easily be integrated in electronic circuits and independently addressed to create the

\section{References:}

[1] H. Ago et al. Phys. Chem. B 103, 8116 (1999).

[2] S. M. Lyth et al. APL 90, 013120 (2007). 